\title{
Analyse théorique du comportement mécanique d'une poutre sandwich NIDA en flexion 3 points
}

\author{
Mohamed Soula ${ }^{1, a}$ Rachid Nasri ${ }^{2}$ et Brahim Rezgui ${ }^{2}$ \\ 1 Unité de recherche génie des matériaux, Département de génie mécanique, École Nationale d'Ingénieurs de Tunis, \\ IPEI El Manar, BP 244, Campus Universitaire, 2092 El Manar1, Tunisie \\ 2 Unité de recherche génie des matériaux, Département de génie mécanique, École Nationale d'Ingénieurs de Tunis, \\ BP 37, Le Belvedère, 1002 Tunis, Tunisie
}

Reçu le 30 avril 2008, accepté le 17 septembre 2008

Résumé - Ce travail présente une étude théorique du comportement mécanique des poutres sandwiches dont le cœur est en nid d'abeilles (NIDA) soumises à un essai de flexion 3 points. Les poutres étudiées sont obtenues à partir de plaques sandwiches destinées à la fabrication de bateaux de plaisance. Deux types de poutres sandwichs sont utilisés. Chaque poutre est composée de deux peaux symétriques dont les fibres sont en verres disposées en mat pour la poutre 1 et en plis croisés pour la poutre 2. L'âme est constituée de feuilles de polyéthylène dont les cellules sont de forme hexagonale. Les modules d'Young longitudinal et transversal des peaux sont obtenus expérimentalement par essai de traction, ils servent au calcul des contraintes normales et de cisaillement. L'effet de la cinématique, de l'élancement (rapport de la longueur de la poutre sandwich par son épaisseur totale), $\frac{L}{h}$, du rapport de l'épaisseur de l'âme par celle de la peau, et de l'effet du rapport du module d'Young longitudinal de la peau $E_{\mathrm{p}}$ par celui de l'âme $E_{\text {a }}$ sur les contraintes normales et de cisaillement sont analysés. Les résultats des contraintes normales et de cisaillement pour trois champs de déplacement différents sont comparés. La différence des résultats justifie les conditions d'utilisation des théories simplifiées de Bernoulli et celle de Timoshenko pour ces types de poutres sandwiches.

Mots clés : Poutre sandwich / NIDA / essai mécanique / flexion 3 points / contraintes

Abstract - Theoretical analysis of the mechanical behavior of a sandwich HONEYCOMB beam under 3 points bending test. This work presents a theoretical study of mechanical behavior of the HONEYCOMB sandwich beams under three point bending load. The studied beams are obtained starting from sandwich plates intended for the manufacture of Yachts. Two types of sandwich beams are used. Each beam is made up of two symmetrical skins of which the fibres out of glasses are laid out in mat for beam 1 and crossed ply for beam 2. The core consists of polyethylene sheets whose cells are of hexagonal form. The longitudinal and transversal Young modulus of the skins are obtained in experiments by tensile tests, they are used for calculation of the normal and shear stresses. The effects of kinematics, the length to the thickness ratio $\frac{L}{h}$, the core to the skin thickness ration and the longitudinal Youngs modulus of the skin $E_{\mathrm{p}}$ to that of the core $E_{\mathrm{a}}$ ratio on the normal and shear stresses are analyzed. Normal and shear stress results for three different displacement fields are compared. The difference of the results validates the conditions of using simplified Bernoulli and Timoshenko theories for these sandwich beams.

Key words: Sandwich beam / honeycomb core / mechanical testing / three point bending / stresses

\section{Introduction}

Les structures sandwiches ont une grande rigidité à la flexion spécifique ce qui représente un des critères de première importance dans le domaine du transport routier, ferroviaire et aéronautique. Cependant, leur applicabilité reste toujours limitée aux structures secondaires.

\footnotetext{
${ }^{a}$ Auteur pour correspondance : Soulamed2003@yahoo.fr
}

En fait, une des principales limitations à leur utilisation est due au manque de connaissance de leur comportement sous diverses sollicitations statique et/ou dynamique en service. De nombreux travaux ont étudié le comportement des structures sandwich dans ces dernières décennies. Di Sciuva et al. [1] ont présenté une théorie modifiée basée sur la théorie de zigzag pour trouver les efforts de cisaillement. Cunningham et White [2] ont mesuré les déformations de cisaillement dans l'âme en NIDA dans 
la poutre sandwich en utilisant des rosettes formées de jauges de déformations collées sur la peau pour une console chargée à l'extrémité libre. Sokolinsky et al. [3] ont étudié le comportement non-linéaire d'une poutre sandwich en utilisant des essais de flexion en 4 points. La poutre sandwich utilisée se compose de peaux en aluminium et d'une âme en mousse de PVC. Les auteurs ont noté que la rupture prématurée de la poutre sandwich avec de faibles couches adhésives est provoquée par des efforts élevés de cisaillement dans la couche supérieure au niveau des interfaces et aux extrémités de la poutre. Huang [4] a développé une méthode de calcul tenant compte de deux couches d'adhésif entre les peaux et l'âme. Il a utilisé un champ de déplacement de premier ordre, modèle de plaque et de poutre, pour étudier la rupture d'un panneau ou d'une poutre sandwich avec un nid d'abeilles de cellules carrées. Rizov [5] a étudié la réponse élasto-plastique des poutres sandwiches avec une âme en mousse soumise à un chargement statique local afin de comprendre les modes de défaillance. Balawi et Abot [6] ont étudié le comportement mécanique des nids d'abeilles hexagonaux par des moyens analytiques. Ils ont établi une corrélation avec des résultats expérimentaux pour les nids d'abeilles en aluminium. Romanoff et al. [7] ont présenté une méthode d'homogénéisation afin d'analyser les contraintes dans une poutre homogène équivalente en chargement statique.

Pagano et al. [12] ont présenté une étude démontrant l'utilité des signes de contraintes notamment de cisaillement dans l'étude du comportement des matériaux composites. Pagano [13] a présenté une solution 3D du problème d'élasticité d'une plaque stratifiée rectangulaire simplement appuyée aux contours pour un nombre arbitraire de couches, il l'a appliquée sur une plaque sandwich.

Dans ce travail on présente une étude expérimentale et analytique permettant d'analyser le comportement mécanique des poutres sandwichs NIDA soumises à un chargement de flexion 3 points. Des essais de traction réalisés sur des éprouvettes découpées à partir des peaux ont permis de déterminer leurs modules d'Young longitudinal et transversal. Ensuite on introduit un champ de déplacement qui tient compte du cisaillement et du gauchissement dans la section droite de la poutre sandwich NIDA en plus d'un champ simplifié qui néglige ses effets. Ces 3 champs sont utilisés afin d'évaluer les contraintes normales et de cisaillement dans la section de la poutre sandwich NIDA au droit de la charge (la plus sollicitée). Les différents effets étudiés sont liés à la cinématique, à l'élancement de la poutre $\left(\frac{L}{h}\right)$, au rapport de l'épaisseur de l'âme et de celle de la peau $\frac{e_{\mathrm{a}}}{e_{\mathrm{p}}}$ ainsi qu'au rapport du module d'Young longitudinal de peau et de celui de l'âme $\frac{E_{\mathrm{p}}}{E_{\mathrm{a}}}$. L'évolution des contraintes normales et de cisaillement dans la section la plus sollicitée de la poutre sandwich en fonction de ces effets est analysée.

\section{Formulation théorique}

Considérons une poutre sandwich (Fig. 1), d'épaisseur totale $h$ et de longueur totale $L$ liée au repère orthonormé



Fig. 1. Poutre sandwich sur deux appuis simples chargée au milieu.

direct $(O, x, y, z)$. Le champ de déplacement en un point $\mathrm{M}(x, y, z)$ est donné par :

$$
\begin{aligned}
& U(\mathrm{M})=\left\{\begin{array}{l}
u(x, z) \\
w(x, z)
\end{array}\right\} \\
= & \left\{\begin{array}{l}
u_{0}(x)-\kappa_{1} z \frac{\partial w_{0}}{\partial x}+\kappa_{2} z \phi_{x}(x)-\kappa_{3} c_{1} z^{3}\left(\phi_{x}(x)+\frac{\partial w_{0}}{\partial x}\right) \\
w_{0}(x)
\end{array}\right\}
\end{aligned}
$$

$x$ est l'abscisse de la section transversale sur la ligne moyenne et $z$ est la cote verticale du point courant par rapport à la ligne moyenne du cœur en NIDA.

Les déplacements $\left(u_{0}, w_{0}\right)$ sont ceux d'un point du plan moyen $(x, y, z=0)$. $\phi_{x}$ est la rotation de la section droite autour de l'axe $y, c_{1}=-\left(\frac{4}{3 h^{2}}\right)$ [8]. Dans le cas où $\kappa_{1}=1, \kappa_{2}=0$ et $\kappa_{3}=0$ le champ de déplacement traduit les hypothèses de Bernoulli. Le cas où $\kappa_{1}=0$, $\kappa_{2}=1$ et $\kappa_{3}=0$ introduit le cisaillement, Mindlin. La prise en compte du gauchissement se traduit dans la théorie de $3^{\mathrm{e}}$ ordre où $\kappa_{1}=0, \kappa_{2}=0$ et $\kappa_{3}=1$. En déformation élastique isotherme, la relation contraintedéformation s'écrit dans le repère de la poutre, comme suit :

$$
\bar{\sigma}_{i j}=\bar{C}_{i j k l} \bar{\varepsilon}_{k l}
$$

Les composantes de $\bar{C}_{i j k l}$ et les déformations associées au champ de déplacement (Éq. (1)) sont développées et données par [9] :

$$
\begin{aligned}
& \varepsilon_{x x}=\varepsilon_{x x}^{(0)}+z \varepsilon_{x x}^{(1)}+z^{2} \varepsilon_{x x}^{(2)}+z^{3} \varepsilon_{x x}^{(3)} \\
& \gamma_{x z}=\gamma_{x z}^{(0)}+z \gamma_{x z}^{(1)}+z^{2} \gamma_{x z}^{(2)}
\end{aligned}
$$

où les déformations sont données dans notre cas comme suit :

$$
\begin{aligned}
\varepsilon_{x x}^{(0)} & =\frac{\partial u_{0}}{\partial x}, \varepsilon_{x x}^{(1)}=-\kappa_{1} \frac{\partial w_{0}}{\partial x}+\kappa_{2} \frac{\partial \phi_{x}}{\partial x}, \varepsilon_{x x}^{(2)} \\
& =0, \varepsilon_{x x}^{(3)}=-\kappa_{3} c_{1}\left(\frac{\partial^{2} w_{0}}{\partial x^{2}}+\frac{\partial \phi_{x}}{\partial x}\right), \gamma_{x z}^{(0)} \\
& =\left(1-\kappa_{1}\right) \frac{\partial w_{0}}{\partial x}-\kappa_{2} \phi_{x}, \gamma_{x z}^{(1)}=0 \operatorname{et} \gamma_{x z}^{(2)} \\
& =-3 \kappa_{3} c_{1}\left(\phi_{x}+\frac{\partial w_{0}}{\partial x}\right)
\end{aligned}
$$

Les relations liant les forces et moments aux déformations sont données par :

$$
\left\{N_{x}, M_{x x}, L_{x x}, P_{x x}\right\}=\int_{h / 2}^{h / 2} \sigma_{x x}\left\{1, z, z^{2}, z^{3}\right\} \mathrm{d} z
$$




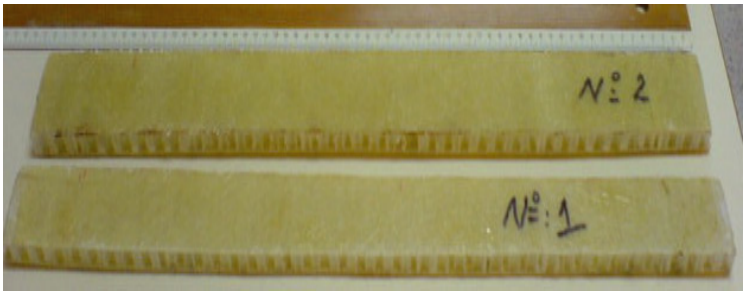

a)

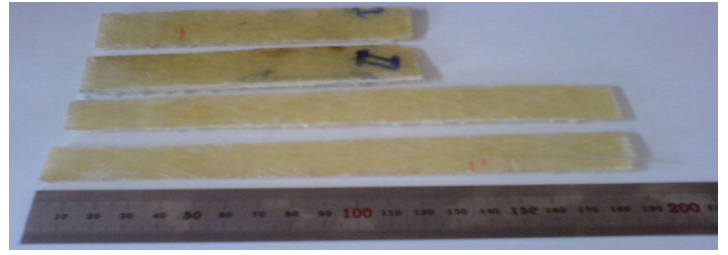

b)

Fig. 2. (a) Les deux poutres sandwiches utilisées. (b) Éprouvettes obtenues à partir des peaux pour la détermination expérimentale des modules d'Young longitudinal et transversal des peaux.

$$
\left\{Q_{x}, R_{x}, S_{x}\right\}=\int_{h / 2}^{h / 2} \sigma_{x x}\left\{1, z, z^{2}\right\} \mathrm{d} z
$$

où $\left(N_{x}, M_{x x}, L_{x x}, P_{x x}\right)$ et $\left(Q_{x}, R_{x}, S_{x}\right)$ sont respectivement les composantes de la force normale, moment fléchissant et les résultantes des contraintes d'ordre supérieur. Ceci permet d'écrire d'après [9] :

$$
\left\{\begin{array}{l}
N_{x} \\
M_{x x} \\
L_{x x} \\
P_{x x}
\end{array}\right\}=\left[\begin{array}{llll}
A_{11} & B_{11} & D_{11} & E_{11} \\
B_{11} & D_{11} & E_{11} & F_{11} \\
D_{11} & E_{11} & F_{11} & G_{11} \\
E_{11} & F_{11} & G_{11} & H_{11}
\end{array}\right]\left\{\begin{array}{c}
\varepsilon_{x}^{(0)} \\
\varepsilon_{x}^{(1)} \\
\varepsilon_{x}^{(2)} \\
\varepsilon_{x}^{(3)}
\end{array}\right\}
$$

et

$$
\begin{gathered}
\left\{\begin{array}{c}
Q_{x} \\
R_{x} \\
S_{x}
\end{array}\right\}=\left[\begin{array}{ccc}
A_{55} & D_{55} & D_{55} \\
A_{55} & D_{55} & D_{55} \\
D_{55} & F_{55} & F_{55}
\end{array}\right]\left\{\begin{array}{c}
\gamma^{(0)} \\
\gamma^{(1)} \\
\gamma^{(2)}
\end{array}\right\} \\
\left\{A_{i j}, B_{i j}, D_{i j}, E_{i j}, F_{i j}, H_{i j}\right\} \\
=\sum_{k=1}^{N} \int_{z_{k}}^{z_{k+1}} \bar{Q}_{i j}^{(k)}\left\{1, z, z^{2}, z^{3}, z^{4}, z^{6}\right\} \mathrm{d} z
\end{gathered}
$$

Les constantes élastiques $\bar{Q}_{i j}^{(k)}$ sont définies pour chaque couche $k$ du composite dans l'annexe A de la référence [9].

Connaissant les vecteurs forces et moments $\left(N_{x}, M_{x x}, L_{x x}, P_{x x}\right)$ et $\left(Q_{x}, R_{x}, S_{x}\right)$ appliqués sur la poutre et les caractéristiques mécaniques on peut calculer les déformations correspondantes à partir des relations (7) et (8). La relation (2) permet de trouver les contraintes $\sigma_{x x}$ et $\sigma_{x z}$.

Les autres contraintes sont obtenues à partir de l'intégration des équations d'équilibre en négligeant les forces de volume :

$$
\begin{aligned}
& \frac{\partial \sigma_{x x}}{\partial x}+\frac{\partial \sigma_{x y}}{\partial y}+\frac{\partial \sigma_{x z}}{\partial z}=0 \\
& \frac{\partial \sigma_{y x}}{\partial x}+\frac{\partial \sigma_{y y}}{\partial y}+\frac{\partial \sigma_{y z}}{\partial z}=0 \\
& \frac{\partial \sigma_{z x}}{\partial x}+\frac{\partial \sigma_{z y}}{\partial y}+\frac{\partial \sigma_{z z}}{\partial z}=0
\end{aligned}
$$

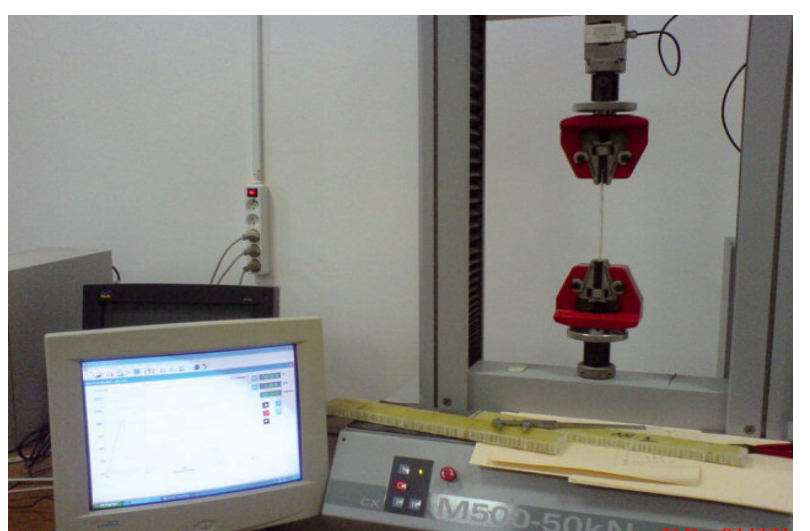

Fig. 3. Dispositif expérimental pour la détermination des modules d'Young longitudinal et transversal des peaux.

La continuité des déplacements entre les inter-couches de la poutre est imposée par les choix de la cinématique équation (1). La contrainte de cisaillement est continue aux interfaces et nulle sur les surfaces libres (Fig. 1).

$$
\begin{gathered}
\sigma_{x z}(z= \pm h / 2)=0 ; \sigma_{z z}(z= \pm h / 2)=0 \\
\sigma_{x z}^{k}\left(x, z_{k+1}\right)=\sigma_{x z}^{k+1}\left(x, z_{k+1}\right) ; \\
\sigma_{z z}^{k}\left(x, z_{k+1}\right)=\sigma_{z z}^{k+1}\left(x, z_{k+1}\right)
\end{gathered}
$$

$k$ est le numéro de couche.

\section{Résultats et discussion}

\subsection{Essai de caractérisation}

Les poutres sandwichs étudiées dans ce papier sont représentées dans la figure 2. Le dispositif expérimental utilisé pour l'obtention des modules d'Young longitudinal et transversal des peaux est représenté dans la figure 3. Les éprouvettes d'essais sont découpées longitudinalement dans la direction des fibres pour la mesure du module d'Young longitudinal et transversalement pour mesurer le module transversal. La machine affiche à chaque fois l'évolution de l'allongement de l'éprouvette en fonction de la charge de traction appliquée. Les valeurs des coefficients de Poisson pour les deux poutres ont été prises dans [11]. Le module de cisaillement est donc calculé à 
Tableau 1. Les caractéristiques géométriques et mécaniques des poutres étudiées.

\begin{tabular}{llll}
\hline & & Poutre $\mathrm{N}^{\circ} 1$ & Poutre $\mathrm{N}^{\circ} 2$ \\
\hline géométrie & longueur $L$ en mm & 417 \\
& largeur $b$ en mm & 44,64 & 56,67 \\
& hauteur peau $e_{\mathrm{p}}(\mathrm{mm})$ & 1,57 & 2,42 \\
& hauteur âme $e_{\mathrm{c}}(\mathrm{mm})$ & 13,68 & 13,68 \\
& hauteur totale $h(\mathrm{~mm})$ & 16,82 & 18,82 \\
& masse $(\mathrm{g})$ & 75,32 & 174,07 \\
& masse volumique $\left(\mathrm{kg} \cdot \mathrm{m}^{-3}\right)$ & 241 & 404 \\
\hline peau & module d'Young longitudinal $E_{x}(\mathrm{GPa})$ & 2,93 & 8,14 \\
& module d'Young transversal $E_{y}(\mathrm{GPa})$ & 2,68 & 7,36 \\
& coefficient de Poisson $\nu_{x y}$ & 0,3 & 0,25 \\
& module de cisaillement $G_{x y}(\mathrm{GPa})$ & 1,10 & 3,13 \\
\hline Âme NIDA & module d'Young longitudinal $E_{x}(\mathrm{GPa})$ & 0,77 & 0,77 \\
& module d'Young transversal $E_{y}(\mathrm{GPa})$ & 0,69 & 0,69 \\
& module d'Young transversal $E_{z}(\mathrm{GPa})$ & 1,45 & 1,45 \\
& coefficient de Poisson $\nu_{x y}$ & 0,85 & 0,85 \\
& coefficient de Poisson $\nu_{x z}$ & 0,15 & 0,15 \\
& coefficient de Poisson $\nu_{y z}$ & 0,15 & 0,15 \\
& module de cisaillement $G_{x y}(\mathrm{GPa})$ & 0,208 & 0,208 \\
& module de cisaillement $G_{x z}(\mathrm{GPa})$ & 0,334 & 0,334 \\
\hline
\end{tabular}

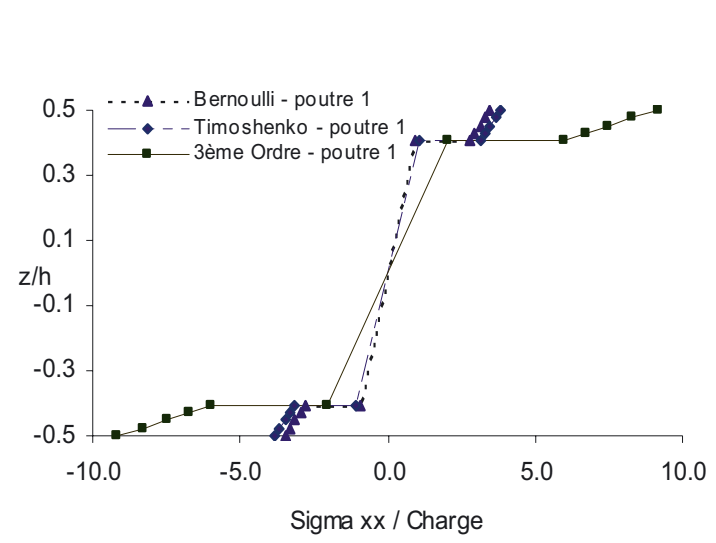

(a)

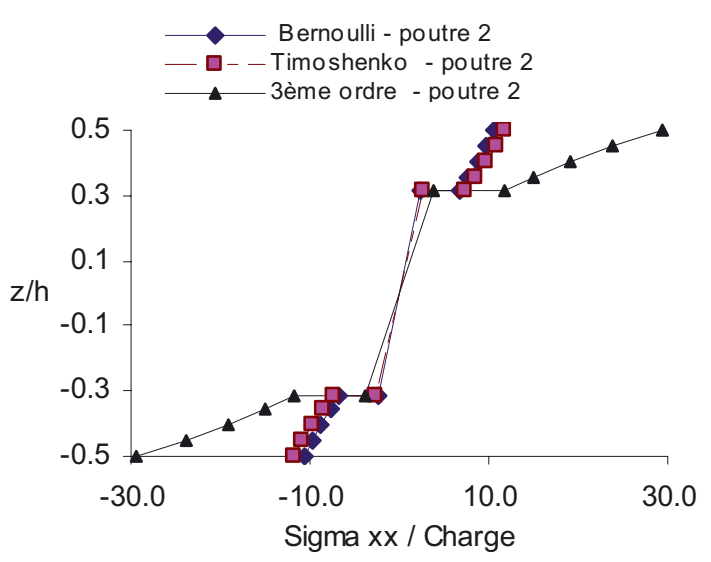

(b)

Fig. 4. Effets de la cinématique sur l'évolution des contraintes normales $\sigma_{x x}$ dans les 3 couches de la poutre sandwich.

partir du module d'Young longitudinal et du coefficient de Poisson.

Pour un matériau isotrope transverse, on a :

$$
G_{x z}=G_{y z} ; \nu_{x z}=\nu_{y z} ; G_{x z}=\frac{E_{x}}{2\left(1+\nu_{x z}\right)} ; G_{x y}=\frac{E_{x}}{2\left(1+\nu_{x y}\right)}
$$

Le tableau 1 donne les caractéristiques géométriques et mécaniques de la poutre sandwich NIDA obtenues par essais pour les peaux et par calcul par homogénéisation pour l'âme.

\subsection{Effet de la cinématique}

Dans ce paragraphe on se propose d'analyser l'influence de la forme du champ de déplacement transversal introduit sur l'évolution des contraintes normales et de cisaillement dans la section transversale de la poutre sandwich au droit de la charge (milieu de la poutre). Pour ce faire, trois théories sont utilisées, celle de Bernoulli [8], celle de Timoshenko [10] et puis la théorie de gauchissement de troisième ordre [8]. Elles sont appliquées aux deux poutres utilisées.

\subsubsection{Contraintes normales}

Les figures $4 \mathrm{a}$ et $4 \mathrm{~b}$ montrent l'évolution de la contrainte normale $\sigma_{x x}$ rapportée à la charge statique pour les poutres 1 et 2 en appliquant les trois théories. Nous constatons, pour les deux poutres qu'au niveau de l'âme en NIDA la théorie de Bernoulli et celle de 


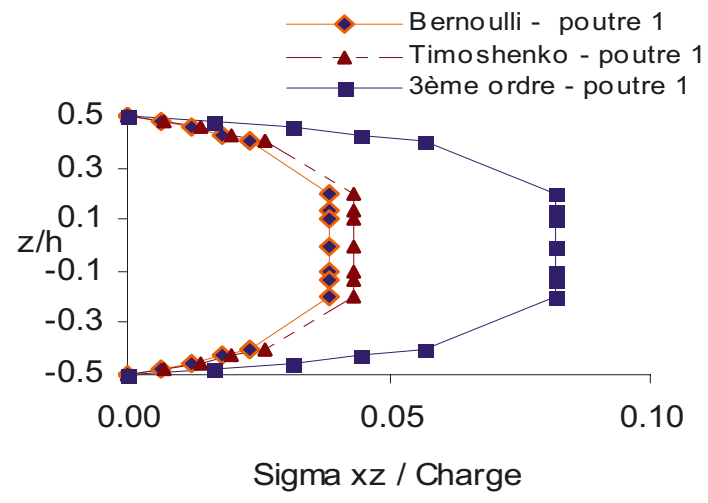

(a)

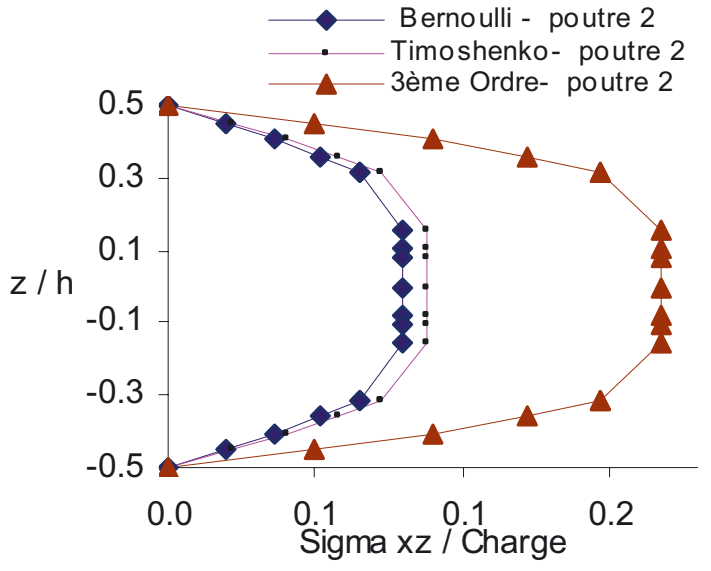

(b)

Fig. 5. Effets de la cinématique sur l'évolution des contraintes de cisaillement $\sigma_{x z}$ dans les 3 couches de la poutre sandwich.

Timoshenko donnent des résultats comparables avec une légère domination de la seconde par effet de cisaillement. Les contraintes obtenues par la troisième théorie qui tient compte du gauchissement sont nettement supérieures aux précédentes (environ 2,4 fois). Ce rapport est pratiquement le même sur toute l'épaisseur de l'âme. Pour la poutre 1, après discontinuité de la contrainte à l'interface, les résultats des deux premières théories s'éloignent légèrement l'un de l'autre tout en respectant un écart relatif constant voisin de $11 \%$ sur toute l'épaisseur de la peau correspondant à leur écart maximal sur l'âme. Le gauchissement augmente la contrainte dans la peau de façon encore plus significative que dans l'âme $(2,4$ pour la première et 2,18 pour la seconde). Toutefois dans la peau de la poutre 2 les deux premières théories donnent des résultats de contraintes normales légèrement plus rapprochés que pour la poutre 1 (10,5\% contre $11 \%$ pour la première). La rigidité de la peau de la seconde poutre par rapport à celle de la première explique cet effet puisque le cisaillement a moins d'effet quand la rigidité augmente. Par contre la prise en compte du gauchissement a un effet similaire pour les deux poutres considérées.

\subsubsection{Contraintes de cisaillement}

Les figures $5 \mathrm{a}$ et $5 \mathrm{~b}$ présentent l'évolution de la contrainte de cisaillement $\sigma_{x z}$ rapportée à la charge statique pour les deux poutres 1 et 2 en fonction des trois théories. Tout comme le cas des contraintes normales dans la peau, la prise en compte du cisaillement n'a pratiquement pas d'influence sur ces contraintes notamment dans le cas de peau rigide (poutre 2). L'écart relatif passe de $16,6 \%$ pour la poutre 1 à $10,5 \%$ pour la poutre 2 .

Dans l'âme en NIDA tout comme pour la contrainte normale, la théorie de Timoshenko donne des contraintes de cisaillement légèrement plus élevées, le rapport est de l'ordre 1. Par contre, la prise en compte du gauchissement augmente notablement les contraintes de cisaillement et le rapport est voisin de 2, pour les deux poutres utilisées.
Le gauchissement fait doubler les contraintes normales et de cisaillement.

\subsubsection{Récapitulation}

La prise en compte du cisaillement augmente les contraintes, nettement moins que celle du gauchissement. Ce dernier phénomène fait croître plus les contraintes normales que les contraintes de cisaillement. La rigidité de la peau favorise l'écart entre les contraintes données par les trois théories notamment au sein de la peau.

\subsection{Effet de l'élancement $\left(\frac{L}{h}\right)$}

Dans ce paragraphe on se propose d'analyser l'influence de l'élancement $\left(\frac{L}{h}\right)$ de la poutre sandwich pour les trois théories et les deux poutres mentionnées ci-dessus.

\subsubsection{Contraintes normales}

Les figures $4 \mathrm{a}-\mathrm{b}$ et $6 \mathrm{a}-\mathrm{d}$ présentent l'évolution de la contrainte normale $\sigma_{x x}$ dans la section du sandwich pour les trois théories utilisées et pour 3 rapports d'élancement de la poutre sandwich $\left(\frac{L}{h}=5,10\right.$ et 50$)$.

On note que, pour la poutre 1 , plus l'élancement est important plus l'écart entre les 3 théories décroît notamment dans l'âme et sur les peaux du côté des interfaces, cet effet est minime aux bords, ce qui veut dire que le gauchissement est plus dominant pour les poutres courtes. En effet le rapport des contraintes passe de 2,55 pour la poutre courte $\left(\frac{L}{h}=10\right)$ à 1,95 pour la poutre longue.

Concernant les deux premières théories utilisées (sans gauchissement) l'écart est très peu décelable notamment dans l'âme en nida. Là aussi l'écart est plus notable pour les poutres courtes (12\% contre $10 \%$ ) ce qui veut dire que l'effet du cisaillement croît quand la poutre est plus courte. 

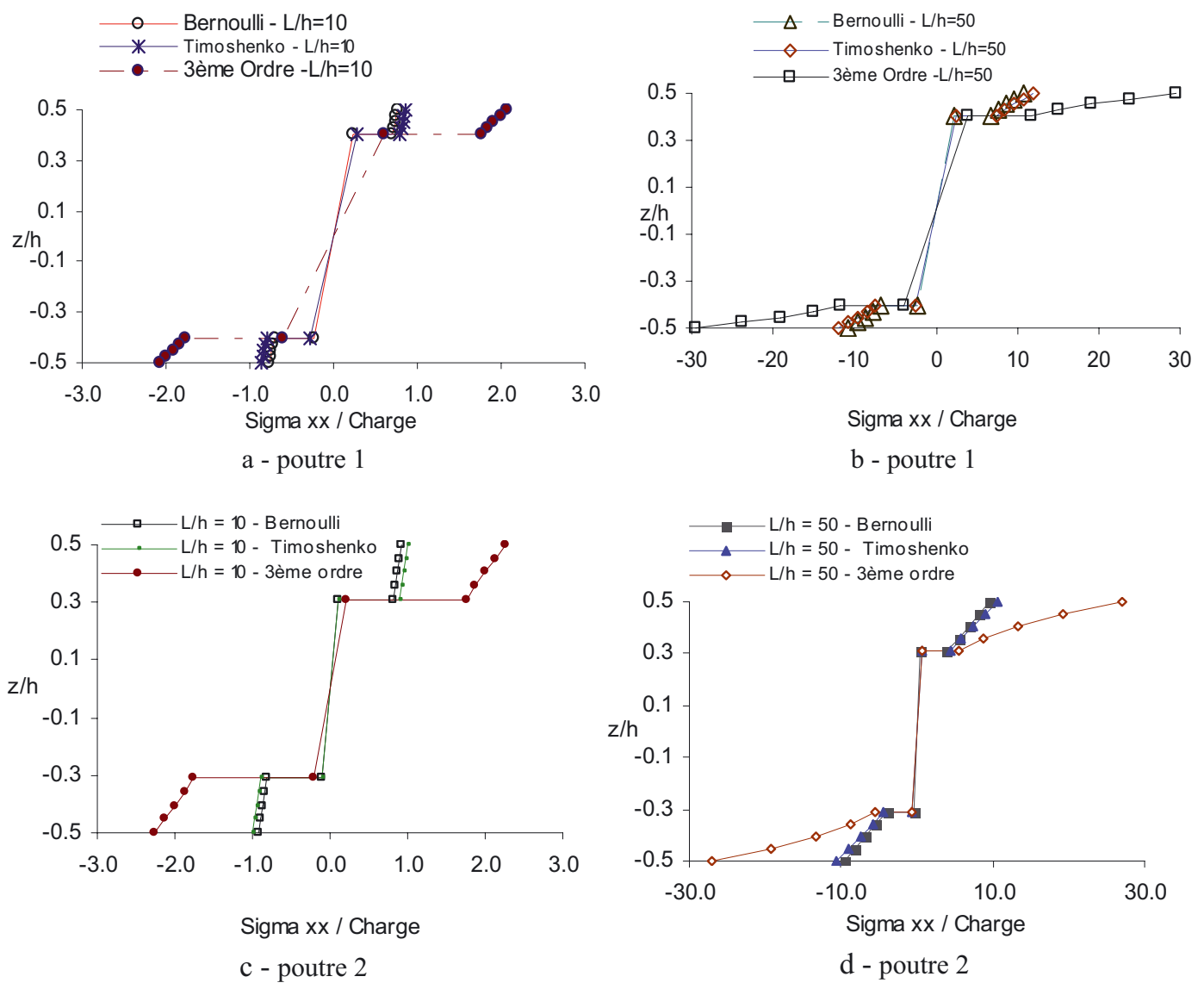

Fig. 6. Effet du rapport $L / h$ sur l'évolution des contraintes normales $\sigma_{x x}$ dans les 3 couches de la poutre 1.

Pour la poutre 2, le décalage introduit par la prise en compte du gauchissement est plus manifeste pour les poutres courtes notamment dans l'âme où on constate étonnamment que les 3 théories coïncident pratiquement quand l'élancement est élevé. La discontinuité introduite par le changement de couche provoque un éloignement des 3 théories et notamment la $3^{\mathrm{e}}$ avec les deux autres au voisinage de l'extrémité et ce même quand l'élancement est fort. Le gauchissement augmente donc la contrainte normale surtout dans la peau et en s'éloignant de la fibre neutre.

\subsubsection{Contraintes de cisaillement}

Les figures $5 \mathrm{a}-\mathrm{b}$ et $7 \mathrm{a}-\mathrm{d}$ montrent l'évolution des contraintes de cisaillement $\sigma_{x z}$ dans la section pour les mêmes élancements que le cas précédant. Dans la poutre 1 plus l'élancement est élevé plus l'écart entre les théories diminue mais ce phénomène est moins prononcé pour la contrainte de cisaillement. En effet le rapport des contraintes dans l'âme est voisin de 2,2 dans les trois situations analysées. Tout de même les deux premières théories se distinguent l'une par rapport à l'autre pour cette contrainte, leur écart relatif passe de $7,7 \%$ pour les poutres longues à $10,5 \%$ pour les poutres courtes. Il est raisonnable que la prise en compte du cisaillement fait monter la contrainte de cisaillement plus notablement que la contrainte normale.

Dans la poutre 2, on constate que les deux premières théories sont plus proches dans la peau qui est plus rigide, que dans la poutre 1 ce qui explique cette légère différence de comportement. L'écart relatif passe de $9 \%$ pour les poutres longues à $18 \%$ pour les poutres courtes. Néanmoins cette différence devient notable dans l'âme de la même manière que pour la première poutre, il s'agit visiblement du même matériau. L'éloignement aussi notable que précédemment au niveau de la théorie de gauchissement aussi bien dans l'âme que dans la peau est pratiquement constant pour les trois élancements étudiés.

\subsubsection{Récapitulation}

Plus l'élancement est important plus les théories de premier ordre peuvent être suffisantes puisque les autres théories donnent des résultats comparables et ce notamment dans la peau pour la contrainte normale.

En effet le gauchissement est très influent pour les poutres courtes au niveau des contraintes normales et tangentielles notamment sur la peau. Par contre pour la contrainte de cisaillement on ne peut pas faire le même constat sauf dans la peau. La prise en compte du cisaillement modifie plus visiblement la contrainte tangentielle mais très peu la contrainte normale. 


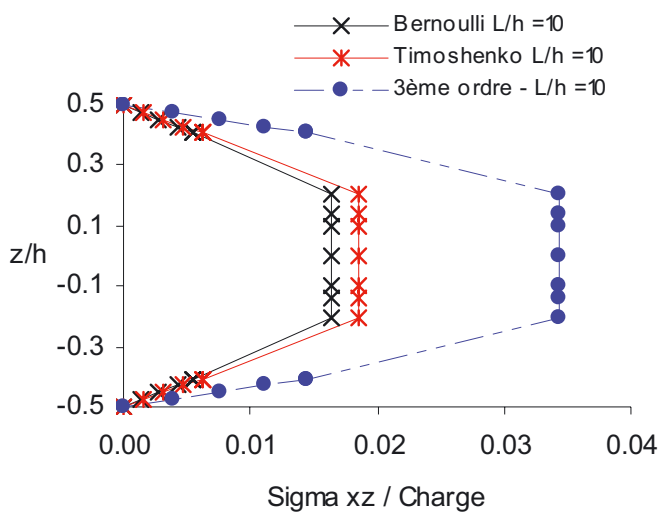

a - poutre 1

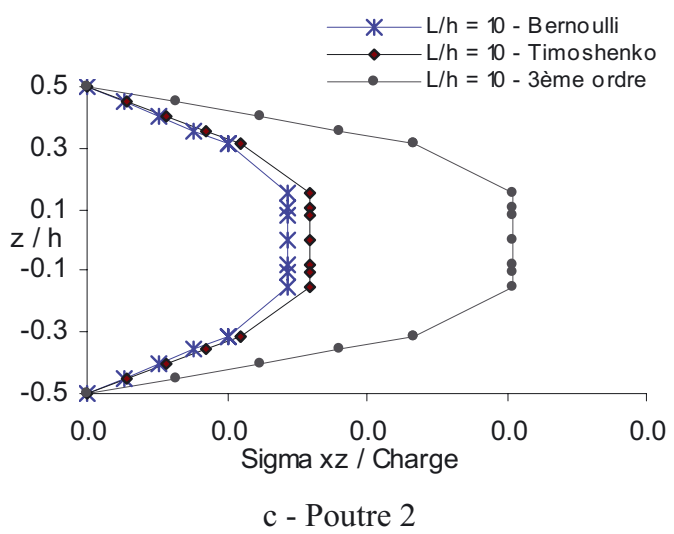

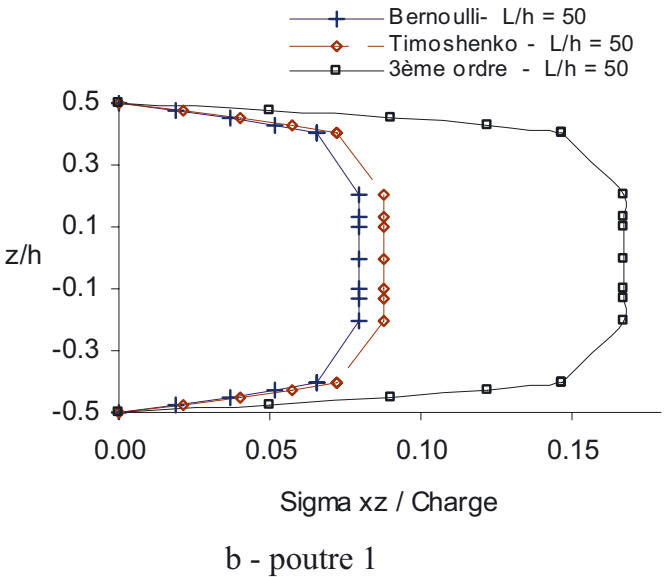

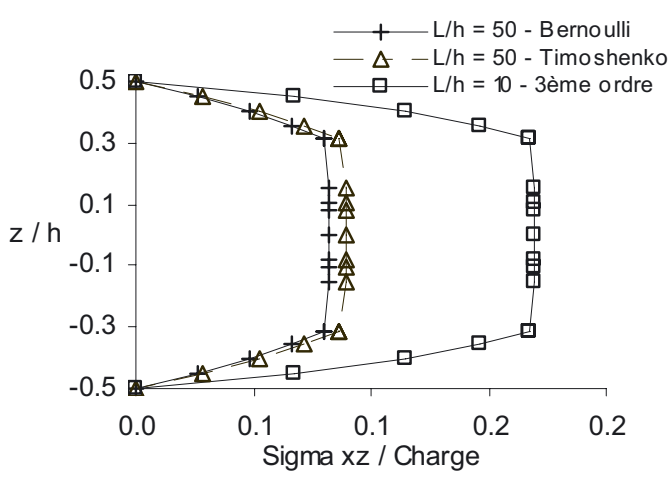

d - Poutre 2

Fig. 7. Effet du rapport $L / h$ sur l'évolution des contraintes de cisaillement $\sigma_{x z}$ dans les 3 couches de la sandwich.

\subsection{Effet du rapport $\frac{e_{a}}{e_{p}}$}

Dans cette section, on se propose d'analyser l'influence du rapport de l'épaisseur de l'âme et de celle de la peau $\frac{e_{\mathrm{a}}}{e_{\mathrm{p}}}$ pour les trois théories appliquées sur la poutre 1 , en gardant la hauteur totale $h$ de la poutre constante.

\subsubsection{Contraintes normales}

Dans les figures $4 \mathrm{a}$, $8 \mathrm{a}$ et $8 \mathrm{~b}$ on se propose d'analyser l'influence sur la contrainte normale $\sigma_{x x}$ des trois théories si le rapport des dimensions peau et âme varie tout en maintenant constante l'épaisseur globale de la section. On constate que si la peau est plus épaisse l'influence du gauchissement diminue dans l'âme, l'écart relatif passe de $128 \%$ à $115 \%$ quand l'épaisseur de la peau double. Par contre cet écart est maintenu constant dans la peau au voisinage de $147 \%$. Toutefois pour les 3 théories, la contrainte normale augmente quand la peau est plus mince cette augmentation est voisine de $45 \%$ dans la peau pour les 3 théories. Par contre dans l'âme l'augmentation de contrainte varie de $57 \%$ pour la théorie de Bernoulli, de $59 \%$ pour la théorie de cisaillement et de $71 \%$ pour celle de gauchissement et ce aussi bien dans l'âme que dans la peau.

\subsubsection{Contraintes de cisaillement}

Les figures $5 \mathrm{a}, 9 \mathrm{a}-\mathrm{c}$ présentent l'influence du rapport de l'épaisseur de la peau et de celle de l'âme sur la contrainte de cisaillement pour les trois théories utilisées. La théorie de gauchissement fait monter la contrainte de cisaillement pratiquement de la même manière pour la peau mince que pour la peau épaisse et ce aussi bien dans la peau que dans l'âme. Quand la peau est plus mince, les contraintes de cisaillement dans l'âme augmentent pour les 3 théories. Leur écart relatif est plus significatif pour la théorie de cisaillement $(2,5 \%)$, il est le moins significatif pour la théorie de gauchissement (1\%). Par contre au niveau de la peau le sens de changement de contraintes est inversé d'une part et il est le même pour les 3 théories d'autre part. On note que cet écart est significatif, il est de l'ordre de $25 \%$ quand l'épaisseur de la peau double.

\subsubsection{Récapitulation}

L'épaisseur relative de la peau par rapport à l'âme a de l'influence aussi bien sur la contrainte normale que sur celle de cisaillement. L'influence du gauchissement est accrue pour la contrainte normale dans l'âme quand l'épaisseur augmente. La contrainte de cisaillement dans la théorie de Timoshenko est la plus touchée par cet effet 


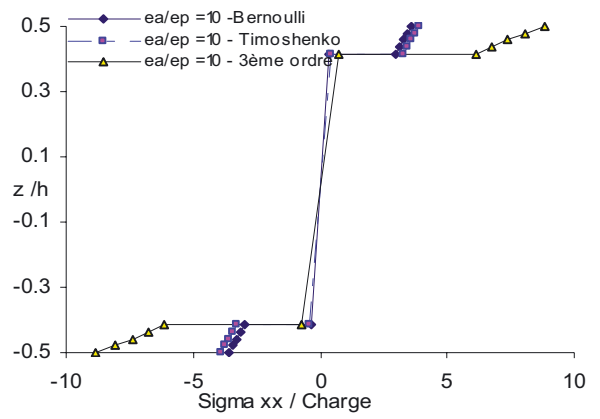

a - Poutre 1



b - Poutre 1

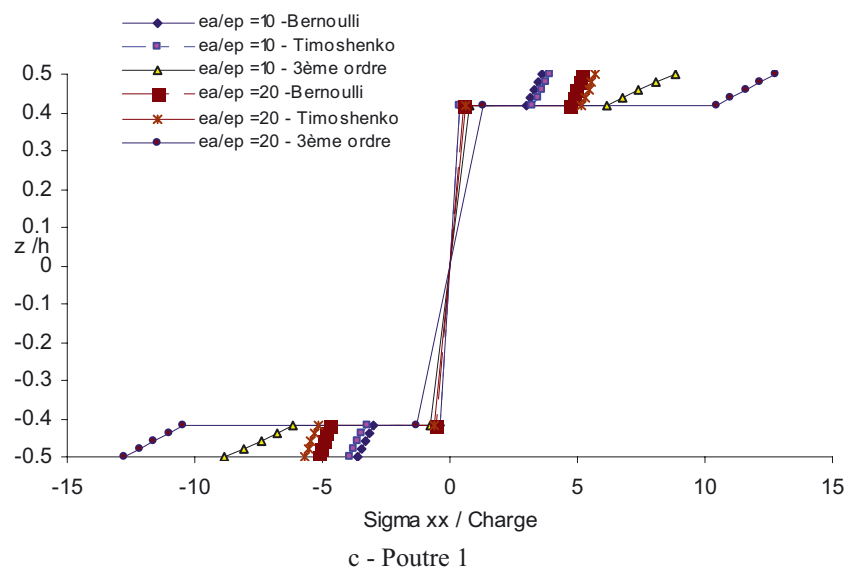

Fig. 8. Effet du rapport $e_{\mathrm{p}} / e_{\mathrm{a}}$ sur l'évolution des contraintes normales $\sigma_{x x}$ dans les 3 couches de la poutre sandwich.

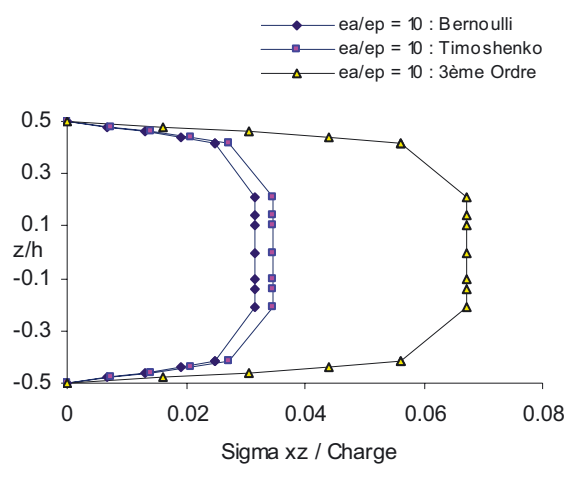

a - Poutre 1

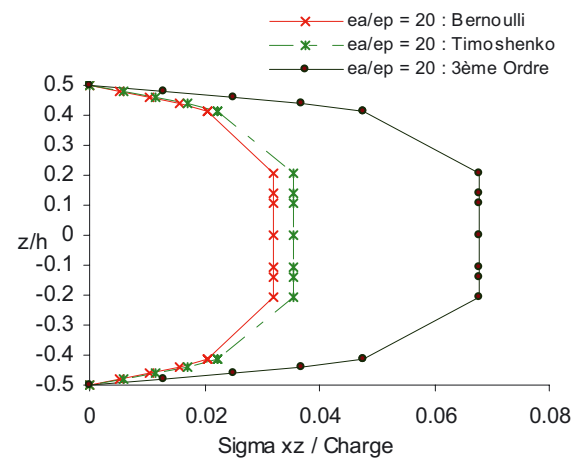

b - Poutre 1

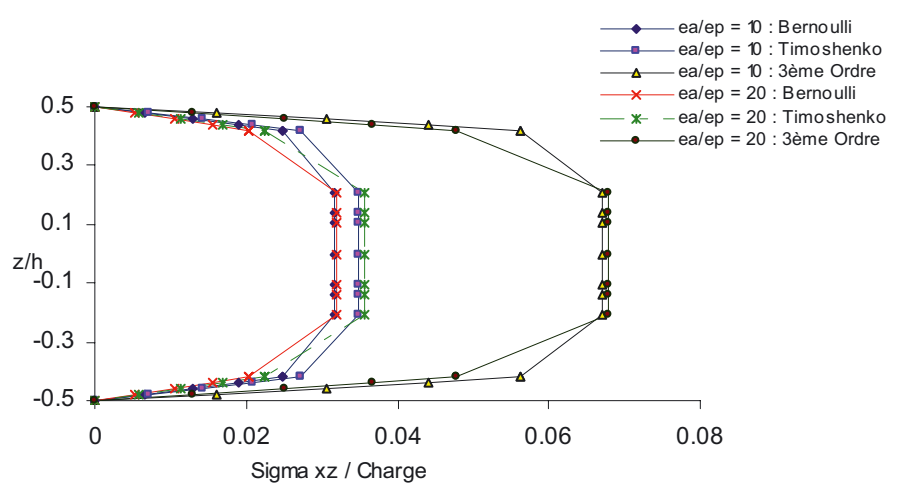

c - Poutre 1

Fig. 9. Effet du rapport $e_{\mathrm{p}} / e_{\mathrm{a}}$ sur l'évolution des contraintes de cisaillement $\sigma_{x z}$ dans les 3 couches de la poutre sandwich. 


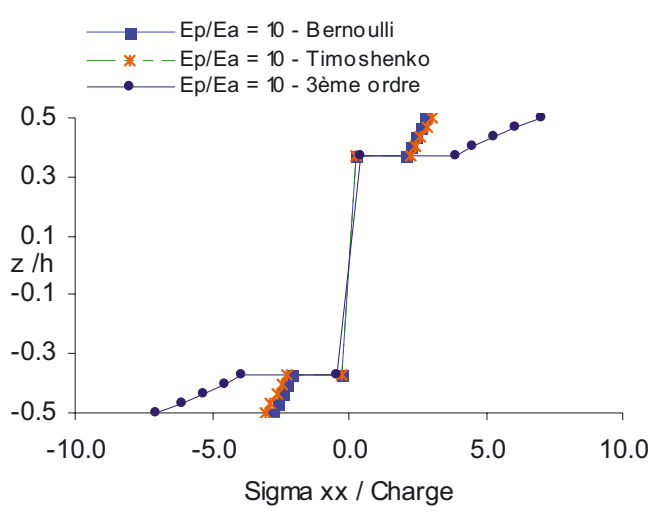

a - Poutre 2

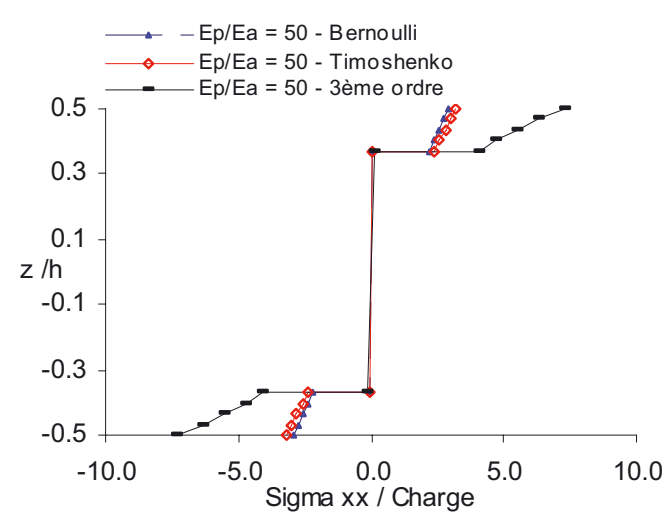

b - Poutre 2

Fig. 10. Effet du rapport $E_{\mathrm{p}} / E_{\mathrm{a}}$ sur l'évolution des contraintes normales $\sigma_{x x}$ dans les 3 couches de la poutre sandwich.



11 a - Poutre 2

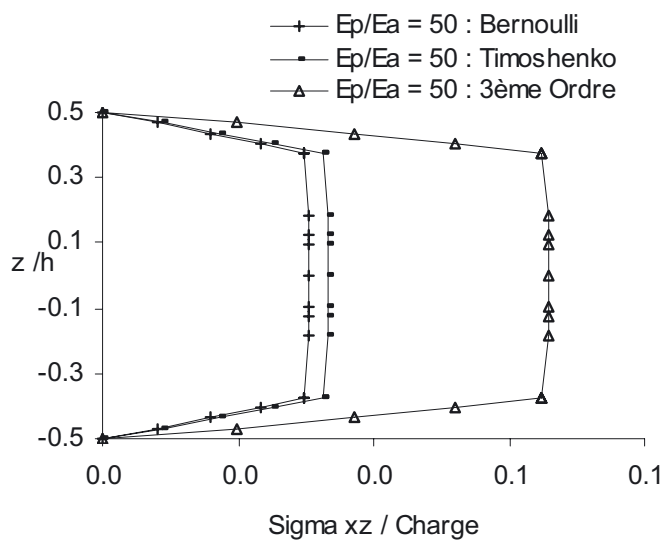

$11 \mathrm{~b}$ - Poutre 2

Fig. 11. Effet du rapport $E_{\mathrm{p}} / E_{\mathrm{a}}$ sur l'évolution des contraintes de cisaillement $\sigma_{x z}$ dans les 3 couches de la poutre sandwich.

d'épaisseur dans l'âme du sandwich. Le sens de variation la contrainte de cisaillement est inversé de l'âme à la peau.

\subsection{Effet du rapport des modules d'élasticité $\frac{E_{\mathrm{p}}}{E_{\mathrm{a}}}$}

Dans cette section on se propose d'étudier l'effet du rapport du module d'Young de la peau et de celui de l'âme $\frac{E_{\mathrm{p}}}{E_{\mathrm{a}}}$ sur l'évolution des contraintes. Pour ce faire on maintient le matériau de la peau ainsi que la géométrie de la poutre 2 puis on agit sur le matériau de l'âme en NIDA.

\subsubsection{Contraintes normales}

Les figures 4b, 10a et 10b présentent l'influence du rapport des modules d'élasticité longitudinale de la peau et de l'âme sur la contrainte normale $\sigma_{x x}$ pour les trois théories utilisées. Il est notable que si le rapport des rigidités augmente, l'influence du gauchissement sur la contrainte normale dans l'âme croît plus lentement pour des rapports très élevés (similaire à une évolution logarithmique). La contrainte normale croît dans la peau et diminue dans l'âme quand le rapport des rigidités est plus élevé pour les 3 théories. L'effet du cisaillement est constant de l'ordre de $9 \%$ pour l'âme et la peau pour les différents rapports de rigidité envisagés. On constate que la variation de contraintes normales dans la peau est plus significative pour les deux premières théories que pour celle de gauchissement $(27 \%$ contre $6 \%)$. Cette variation est plus faible pour des rapports de rigidités plus importants (évolution logarithmique). Contrairement au cas précédent et dans l'âme, le rapport de rigidité a une influence beaucoup plus notable pour les 3 théories. La variation de la contrainte est de $286 \%$ pour les deux premières théories et de $316 \%$ pour celle de gauchissement quand le rapport est multiplié par 5 . Pour des rapports plus élevés, la croissance de contraintes est accélérée.

\subsubsection{Contraintes de cisaillement}

Les figures 5b, $11 \mathrm{a}$ et $11 \mathrm{~b}$ sont similaires aux figures $4 \mathrm{~b}, 10 \mathrm{a}$ et $10 \mathrm{~b}$ mais relativement à la contrainte de cisaillement $\sigma_{x z}$. L'augmentation du rapport de rigidité fait monter la contrainte dans la peau contrairement à celles dans l'âme. L'effet du cisaillement diminue quand le rapport des rigidités augmente aussi bien dans la peau 
que dans l'âme. Cette baisse est plus notable dans l'âme où son évolution avec le rapport de rigidités est plus rapide. L'effet du gauchissement croît avec le rapport de rigidités dans l'âme mais il décroît dans la peau. L'augmentation du rapport de rigidités est le plus important pour la théorie de cisaillement $(17 \%)$ et il est le plus faible pour la théorie de gauchissement (11\%) aussi bien dans la peau que dans l'âme. En augmentant le rapport de rigidités, cette variation diminue (évolution logarithmique) pour la peau. Dans l'âme, cette diminution est beaucoup plus significative pour la théorie de cisaillement où la contrainte se stabilise avec l'évolution du rapport de rigidités.

\subsubsection{Récapitulation}

Le rapport de rigidités a une influence très significative pour toutes les théories utilisées aussi bien en contraintes normales que de cisaillement dans la peau et l'âme de la poutre sandwich. La contrainte normale obtenue par la théorie de gauchissement est plus sensible à ce rapport dans l'âme. Par contre les deux autres théories sont plus touchées par ce rapport dans la peau. L'augmentation du rapport de rigidités fait monter la contrainte de cisaillement dans la peau et il la fait baisser dans l'âme.

\section{Conclusions}

Des essais de traction sur des éprouvettes taillées à partir des peaux ont été réalisés. Les modules d'Young longitudinal et transversal des peaux ont été mesurés. Les caractéristiques mécaniques de l'âme sont obtenues par un calcul d'homogénéisation. Une étude théorique tenant en compte le cisaillement et le gauchissement a été réalisée. Les effets de ces théories sur la variation des contraintes normales et de cisaillement dans la poutre sandwich ont été analysés. Pour toutes les caractéristiques géométriques et mécaniques du sandwich utilisé l'effet du gauchissement fait croître les contraintes normales et de cisaillement. Pour une seule situation les contraintes normales données par les 3 théories sont semblables dans le corps en NIDA. Il s'agit du cas d'une peau très rigide où l'âme fonctionne comme un ressort où les contraintes sont minimes et la répartition des contraintes normales est pratiquement linéaire. Par contre la répartition des contraintes de cisaillement est peu sensible à la disproportion des rigidités. L'effet de la prise en compte du cisaillement augmente la contrainte de cisaillement dans l'âme alors que son effet sur la peau reste minime. Plus la poutre est élancée plus le gauchissement perd de l'influence sur les contraintes. Les peaux minces génèrent des contraintes de cisaillement plus fortes dans l'âme et moins fortes dans la peau. L'amincissement de cette peau accroît l'effet du gauchissement dans l'âme sans influence significative dans la peau. La rigidification des peaux fait monter les contraintes normales et tangentielles dans l'âme nettement plus que dans la peau. Le phénomène de gauchissement est influencé par l'augmentation de cette rigidité notamment dans l'âme. Les résultats obtenus permettront entre autres l'optimisation du choix des structures sandwichs pour la fabrication de certaines pièces industrielles. Des mesures en cours de contraintes donneront sûrement plus de garantie à ces résultats et à leur limitation selon le choix des matériaux.

Remerciements. Les auteurs remercient l'entreprise TRIMEX-SARL pour son soutien et l'intérêt apporté à ce travail et les lecteurs pour leurs suggestions et remarques.

\section{Références}

[1] M. DiSciuva, U. Icardi, E. Miraldi, G. Ruvinetti, Holographic interferometry assessment of stress distribution in sandwich beams in bending, Composites Part B: Eng. 32 (2001) 175-184

[2] P.R. Cunningham, R.G. White, A new measurement technique for the estimation of core shear strain in closed sandwich structures, Compos. Struct. 51 (2001) 319-334

[3] V.S. Sokolinsky, H. Shen, L. Vaikhanski, S.R. Nutt, Experimental and analytical study of nonlinear bending response of sandwich beams, Compos. Struct. 60 (2003) 219-229

[4] S.J. Huang, An analytical method for calculating the stress and strain in adhesive layers in sandwich beams, Compos. Struct. 60 (2003) 105-114

[5] V.I. Rizov, Non-linear indentation behavior of foam core sandwich composite materials-A 2D approach, Comp. Mat. Sci. 35 (2006) 107-115

[6] S. Balawi, J.L. Abot, The effect of honeycomb relative density on its effective in-plane elastic moduli: An experimental study, Compos. Struct. (2007) doi : 10.1016/j.compstruct.2007.08.009

[7] J. Romanoff, P. Varsta, A. Klanac, Stress analysis of homogenized web-core sandwich beams, Compos. Struct. 79 (2007) 411-422

[8] J.N. Reddy, Mechanics of laminated composite plates, CRC Press, 1997

[9] M. Soula, R. Nasri, A. Ghazel, Y. Chevalier, The effects of kinematic model approximations on natural frequencies and modal damping of laminated composite plates, J. Sound Vib. 297 (2006) 315-328

[10] S.P. Timoshenko, On the correction for shear of differential equation for transverse vibration of prismatic bars, Philos. Mag. Ser. 41 (1921) 744-746

[11] L.F. Nielsen, Composite Materials Properties as Influenced by Phase Geometry, Springer-Verlag, Berlin Heidelberg, 2005, printed in the Netherlands

[12] N.J. Pagano, P.C. Chou, The Importance of Signs of Shear, Stress and Shear Strain in Composites, J. Composite Materials 3 (1969) 166-172

[13] N.J. Pagano, Exact solutions for rectangular bidirectional composites and sandwich plates, J. Composite Materials 4 (1970) 20-34 